\title{
IMMUNITY AND STRONGYLOIDIASIS*
}

\author{
Domingos de Paola e Kalil Madi
}

The pathologic picture in strongyloidiasis depends on a variety of factors. The pathogenic action of the worm varies in intensity and type according to the stage of parasitism, in terms of the various phases of the evolutive cycle and especially to the auto-infection cycle. Attention must be given to factors within the host organism, such as the state of nutrition and the various associated and infectious conditions.

The contrast between the broad geographical distribution of this parasitosis in its asymptomatic or minor form, and the samall frequency of fatal cases leads on to suppose that the severe forms do not depend exclusively on a greater aggressivity of the worm which according to Faust $^{3}$, in 1935 - is becoming progressively adapted to parasitic life. Conditions displayed by the host such as malnutrition or impairment of the immune defenses are probably the prime factors through which the fatal course of the parasitosis in unleashed. The description of the socalled hyperinfective forms in human cases associated with the use of corticosteroids, debilitating or malignant diseases with immunological deficiencies has made it very clear the evident immunological implications of this helminthiasis. If this is not so, it is hard to account for the existence of longstanding infections which have had no significant effects on the host. It is nevertheless true that malnutrition may be caused by the slow progressive deprivation provoked by the nematode, thougdh it may also arise from associated morbid states. This is very true in our infantile cases. In fact Hartz, in 1954 , stated that auto-infection followed by unfavourable evolution of the disease ocurred only in individuals whose health has been undermin. ed. In view of this vicious cycle and knowing that several intestinal lesions may be due to both malnutrition and parasitism, we think that this and other similar diseases as belonging to a morbid complex of malnutrition and infection. The synergism is very clear and in infants we rarely see one without another. The immunological problems belonging to this morbid complex has been well established, producing severe thymic atrophy similar to those described in several congenital immunological deficienses ${ }^{6}$.

Reviewing the cases recorded in the last 30 years in literature and those observed by us, in about 80 cases, we believe it is possible to describe three clinic pathological forms in which the disease occurs. It must be said however that the infection has a chronic character, although the worse lesions present chronic and acute changes, and that sometimes it is very difficult to separate what is due to the disease and what is due to complications.

\section{1 - MINOR FORMS}

These correspond to a catarrhal enteritis seen in patients where the causa mortis is produced by other factors or agents, related or not to the gut lesions, or from biopsy cases. The structure of the intestine is not substantially modified,

* From the Department of Pathology, Federal University of Rio de Janeiro and Conselho Nacional de Desenvolvimento Científico e Tecnológico - CNPq. 
showing cases with a mucoid exsudate in duodenal and jejunal mucosa, conges. tion or, when the case does not show signs of acute irritation, a simple and fine granular aspect of the mucous membrane, with some flattening of folds. This is called granular enteritis. Microscopically the catarrhal involvement shows congestion, diffuse eosinophilic infiltration and increase of the mononuclear cells in the lamina propria, the worms and eggs occupying the crypts. The epithelial barrier, except for signs of regeneration, seems well preserved. The granular enteritis is due to chronic inflamation, epithelial regeneration with a decrease of goblet cells. The villi may have a discrete reduction in height and increase in thickness. In these forms the affection keeps a segmentary topography and does not go far beyond the muscularis mucosae.

\section{2 - MODERATE FORMS}

Here the signs of irritation and incrimination of the gut lesions in the causa mortis are evident. The mucosa is coarsely granular, with small ulcerations or punctate hemorrhages, the folds may not be seen and the submucosa is conspicuously edematous giving, sometimes, an appearance of the brain circumvolutions to the surface. Histologically, besides the presence of the worms in the surface, signs of penetration of larvae with edema and acute inflamation, granulation tissue and granulomatous reaction may be seen around died larvae in the submucosa. The mucosa is destroyed by small areas of necrosis or abccess, some of them with giant ceils around the worms, or small ulcerations which do not expose the submucosa extensively. The mucosa shows no goblet cells but irregular regenerative epithelium, with sometimes pseudoestratified areas. Here the villi present the typical changes of diffuse atrophy (sprue-like pattern), with or without the presence of worms. In these forms there are no significant involvement of the large intestine.

\section{3 - SEVERE FORMS}

The character of these forms is that of an extensive ulcerative enteritis, with rigidity of the wall by a mix of edema and granulation tissue, chronic and acute inflamation, with disssemination of larvae beyound the submucosa and the proximal small intestine, with edematous, hemorragic or ulcerative forms of colitis. There is also a necrotizing form affecting both bowels, but to us they look as associated bacterial or is. chaemic lesions. The mucous membrane - when seen in areas without ulceration or necrosis - shows intense atrophy; the ileum and colon present almost no mucus or globet cells; regeneration is a common finding in these areas. Besides, necrosis may extend to deeper layers, there is flegmonous inflamation mixed with granulomatous reaction around the larvae seen from mucosa to serosa. The characteristic lesion which suggests the way in which the worms disseminate is a granulomatous endolymphangitis. This lesion evidenced by $\mathrm{Hartz}^{4}$ in 1946, is of great importance in the aggravation of the local process because it leads to lymphatic obstruction which, with ede$m a$, is partially responsible for hypotonia of the small intestine and for malabsorptive changes seen clinically. We think that dissemination may also be through the veins because we must say that edematous thickening of submucosa, so sensitive to hemodynamic modifications, is due both to inflamation and stasis. The cases with involvement of the large gut where a great number of larvae is seen in the submucosa without adult worms or eggs present in the mucosa, are particularly interesting because they suggest penetration far from their natural habitat (duodenum and initial jejunum) and probably without the outside cycle.

As we have stated, several factors are involved in the production of lesions by Strongyloides stercoralis. Experimentation - possibly of greater scope rather than human pathology - has provided data clarifying the mechanisms whereby Strongyloides stercoralis attacks the host organism. Faust, ${ }^{3}$ in 1935 , felt that the parasite had both traumatic and lytic actions. The traumatic action was believed to be the result of the presence of females in the glands of the mucous membrane, causing destruction of the 
grandular structures. This mechanism seemed to be reinforced by the directly lytic actions of the worm on the epithelial cells. One will understand better these damaging actions if it is done in a mucosa with the atrophic changes pro. duced by malnutrition ${ }^{6}$. Such a mechanism of action accounts for the appearance of catarrhal enteritis, as the sim. plest form of elimination of the worm. We agree with Faust, that the minute mucosal lesions may expose the organ to penetration of bacteria, which then would cause necrosis and ulceration. The segmentary distribution of the le. sions argues in favour of the direct action of parasite: worms, and eggs are mainly found in duodenum and initial jejunum.

With the purpose of studyng the synergism between malnutrition and parasitism, several atempts have been made. First we have associated proteincaloric defficiency with infection by Strongyloides ratti, a non-offensive worm for the rat. The results have shown almost no lesions in undernourished rats and both controls lad libitum and isocaloric), but the undernourished animals had an accentuated delay on the elimination of worms. Another experiment in collaboration with Ogilvie and Tomkins, from London, associating malnutrition and Nippostrongy/us brasiliensis infecction, has shown smilar but better results: undernourished had very low titters of seric $\lg E$, almost no eosinophils and mast cells in the gut lamina própria after a second infection, and did not eliminate the worms of both infections. This is particularly important, for according to Ogilvie and Parrot $^{8}$ (1977), in the infection by nematodes there is an increase in seric $\mathrm{lg} \mathrm{E}$ and eosinophils and mast cells in the lamina propria. This, however, does not seem to be the decisive immunological fact in the expulsion of worms and indeed Ogilvie and Parrot have reported a two-step mechanism in which $\mathrm{lgG}$ antibodies damage the worms which are then expelled by sensitized, non-imunoglobulin bearing lynphocytes (" $T$ " cells). The first step is present in lactating and young rats; but these animais do not eliminate Nyppostrogylus brasiliensis, this happens only after the 7 th or 8 th week of age in the normal animal when their " $T$ " cell system is then functioning. We think that undernourished animals have a pathological state different from a simple delay or decrease in their development, because both reactions are altered when they are infected with Nyppostrogylus brasiliensis. But as we have already stated, other factores must be considered because the created situation is of a morbid complex, nothing being specific.

Another change that should be emphasized is the blockage of immunoblasts towards the gut lamina propria. As described by Ogilvie and Parrot ${ }^{8}$ (1977), the most effective cells involved in worm expulsion come from the mesenteric lymphonodes and toracic duct. We wonder to what extent the granulomatous lymphangitis could produce blockage towards the traffic of the immunoblasts. Besides the local lesion, something must be said about the ways in which the parasite may control the host's capacity to respond immunologically. When rats are infected with Trhichinella spiralis, their ability to respond to urelated antigens is greatly reduced. Faubert and Tanner ${ }^{2}$ have presented evidence that the larvae of Thichinella spiralis (but not the adult worms or the muscle stage larvae) secrete a factor which destroys lumphocytes. It is clear from this and other invesiigations that parasites have a marked effect on the ability of their hosts to respond immunologically and this is a major factor underlyng their long persistence in their hosts. Would these informations, in view of the severe pathological changes see in human cases, allow us to think of an immune paralysis in strongyloidiasis? 


\section{REFERENCES}

1. DE PAOLA, D.; DIAS, L.B. \& RODRIGUES DA SILVA, J.: "Enteritis due to Strongyloides stercoralis: a report of 5 fatal cases". Amer. J. Dig. Dis., 7: 1086, 1962.

2. FAUBERT, G.M. \&TANNER, C.E.: "Leucoaglutination and cytotoxicity of the serum of infected mice and of extracts of Trichinella spirallis and the capacity of infected mouse sera to prolong skim allografts" Immunology, 28: 1041. 1975.

3. FAUST, E.C.: "Experimental studies on human and primate species of Strongyloides. IV. The Pathology of Strongyloides infections" A.M.A. Arch. Path., 19:769, 1935.

4. HARTZ, P.H.: "Human strongyloidiasis wich internal auto-infection" A.M.A. Arch. Path., 42:601, 1946.
5. HARTZ, P.H.: "Strongyloides with internal auto-infection in children" Docum. Med. Geogr. et Trop., 6:61, 1954 and Trop. Dis. Bull., 51 : 956, 1954.

6. MADI, K.; JERVIS, H.; ANDERSON, P.R. \& ZIMMERMANN, M.R.: "A protein defficient diet: effect on liver, pancreas, stomach and small intestine of the rat" A.M.A. Arch. Pat., 89: 38, 1970.

7. MADI, K. \& DE PAOLA, D.: “Deficiências Nutricionais'. In Mecanismos Básicos de Doença, Ed. por De Paola, D.; Livraria ATHENEU, Rio, 1977.

8. OGILVIE, B.M. \& PARROT, D.M.Y.: "The immunological consequences of nematode infection". In Immunology of the Gut. Ciba Foundation Symposium, 46, 183-201, 1977. E/sevier/Excerpta Medica/North-Holland. 